\title{
Obtención y caracterización de materiales adsorbentes a partir de cascarilla de arroz
}

\author{
Production and Characterization of Adsorbent Materials from Rice Husk
}

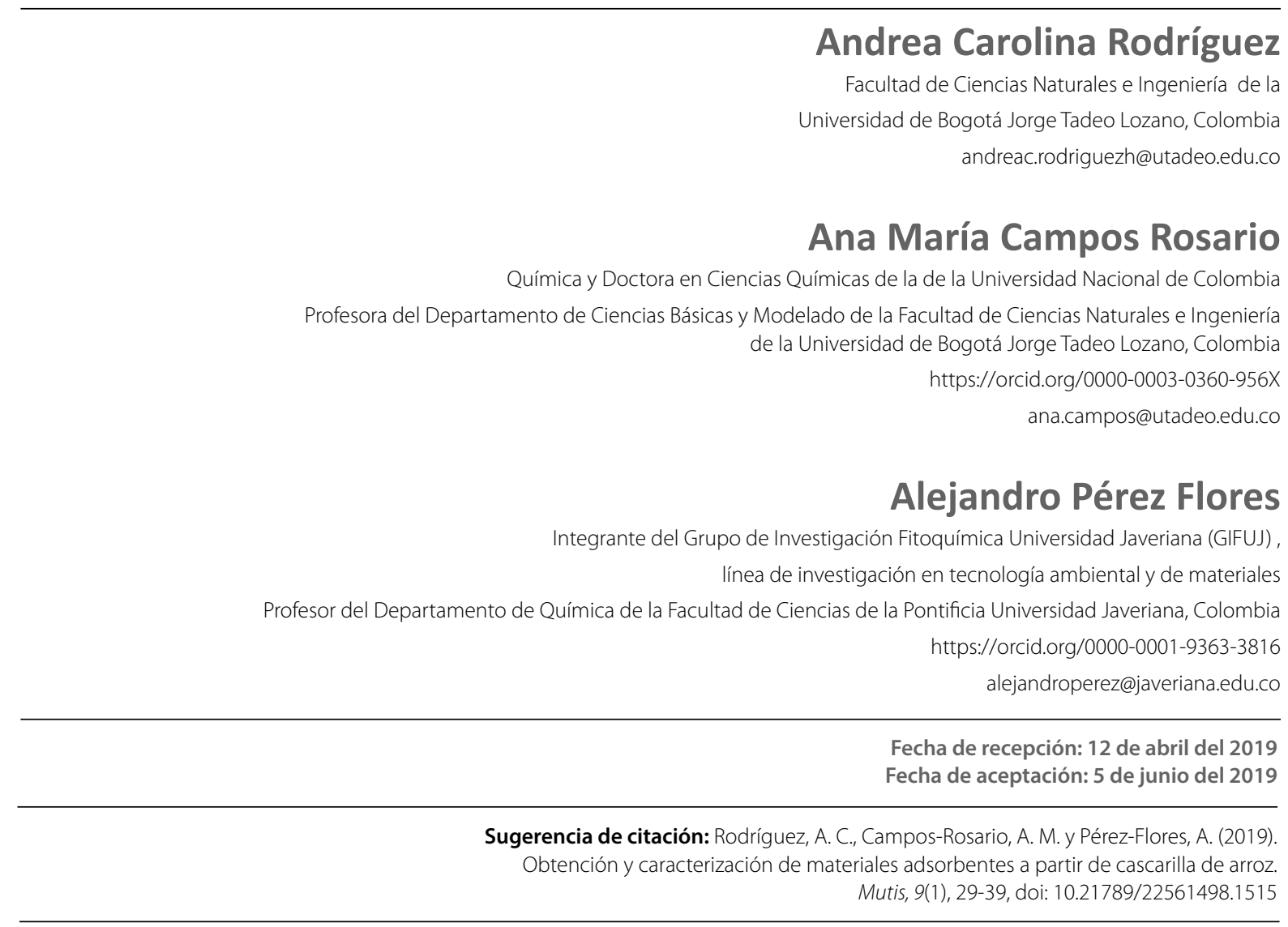

\section{RESUMEN}

La utilización de residuos agroindustriales para la producción de materiales con cualidades específicas es de importancia para solucionar algunos problemas ambientales. La cascarilla de arroz, un desecho agrícola abundante en Colombia, podría ser utilizada como un adsorbente de bajo costo para moléculas como colorantes o metales pesados presentes en las corrientes de efluentes. Este trabajo de investigación presenta la obtención y caracterización de materiales adsorbentes a partir de cascarilla de arroz, producidos mediante activación química con ácido fosfórico. Las propiedades físico-químicas de los materiales fueron evaluadas por medio de diferentes técnicas de caracterización que incluyeron análisis próximo, determinación del número de yodo, adsorción de azul de metileno, adsorción-desorción de nitrógeno, análisis de los grupos funcionales presentes en la superficie del adsorbente a través de la espectroscopia de infrarrojo (FTIR), así como un análisis de la morfología de las cenizas mediante microscopia electrónica de barrido (SEM). Los resultados indican que las cenizas producidas tienen un 
área superficial aproximada de $320 \mathrm{~m}^{2} / \mathrm{g}$, la cual disminuye directamente dependiendo de la cantidad de ácido usado en la activación. Los tamaños de poro de las muestras trabajadas oscilan entre 4 y $12 \mathrm{~nm}$, lo cual indica que se obtuvieron sólidos mesoporosos. Respecto a la capacidad de adsorción, los resultados muestran que el proceso de producción de las cenizas activadas a partir de cascarilla de arroz como materiales adsorbentes es viable, mostrando así una nueva oportunidad de aprovechamiento para los materiales considerados como desechos agrícolas.

Palabras clave: cenizas, cascarilla de arroz, activación química, adsorción, azul de metileno.

\section{ABSTRACT}

Agricultural waste management for the production of materials with specific uses is important for the remediation of certain environmental problems. Rice husk, an abundantly agricultural waste in Colombia, could be a low cost adsorbent for molecules such as dyes or heavy metals in effluent streams. In this research, we address the production and characterization of rice husk ash obtained by chemical activation with phosphoric acid as an adsorbent material. The physical-chemical properties of the materials were evaluated using different characterization techniques, such as close analysis, iodine number, adsorption of methylene blue, adsorption-desorption of nitrogen, analysis of the functional groups on the surface of the adsorbent through infrared spectroscopy (FTIR), as well as an analysis of the morphology with scanning electron microscopy (SEM). The materials have a surface area around $320 \mathrm{~m}^{2} / \mathrm{g}$, which decreases depending on the amount of acid used in the activation. The pore size in the studied samples ranged between 4 and $12 \mathrm{~nm}$, which suggest that mesoporous solids were obtained. Regarding the adsorption of methylene blue, results show that the production of the activated ashes from rice husk as adsorbent materials is viable, presenting a new opportunity for the use of materials considered as agricultural waste.

Keywords: Rice husk ash, biomass, chemical activation, adsorption, methylene blue.

\section{INTRODUCCIÓN}

En Colombia, uno de los principales residuos agroindustriales es la cascarilla de arroz (CA), puesto que, después del café y el maíz, el cultivo de arroz ocupa el tercer puesto respecto a la cantidad de cosecha generada, con un porcentaje de $13 \%$. No obstante, el $30 \%$ del peso total del residuo de CA es usado generalmente como combustible o sustrato (Areiza-Segura, 2012; Carrillo-Quijano, Albarracín-Caballero, \& Pereira-Hernández, 2013; Federación Nacional de Industriales del Arroz, 2013).

La CA en un residuo importante para la obtención de subproductos con valor agregado, teniendo en cuenta su composición: lignina (22,80\%), celulosa $(39,05 \%)$ y otros componentes, dentro de los cuales se hallan principalmente compuestos orgánicos, como proteínas (3,56\%), extractos no nitrogenados (6,60\%) y extractos con éter (0,93\%) (Martínez-Angel, Pineda-Vásquez, López-Zapata, \& Betancur-Vélez, 2010; Valverde, Sarria, \& Monteaguido, 2007). Además, la composición química aproximada de la CA incinerada es: carbono fijo $(16,67 \%)$, cenizas $(17,89 \%)$ y volátiles $(65,47 \%)$. Por otro parte, dentro de las propiedades físicas más relevantes de la CA se encuentra su baja densidad, por lo cual requiere grandes espacios para su almacenamiento. Además, este material presenta ciertas características fisicoquímicas que dificultan su biodegradación, haciendo que se convierta en un gran contaminante (Prada \& Cortés, 2010; Quinceno-Villada \& Mosquera-Gutiérrez, 2010, Sierra-Aguilar, 2009). Con base en lo anterior, se hace necesario investigar otras posibles aplicaciones y/o la obtención de nuevos productos a partir de $\mathrm{CA}$, puesto que en la mayoría de los casos se corre el riesgo de que este material no sea dispuesto de manera adecuada, ocasionando así problemas ambientales relacionados con la dispersión de polvo y cascarilla.

Las cenizas, al tener propiedades similares a los carbones, son utilizadas en diversos campos, tales como la decoloración de bebidas, la adsorción de vapores generados a partir de procesos carboquímicos, la separación de compuestos en fase líquida y gaseosa y la purificación de aguas, entre otros (Klose, Rincón, \& Gómez, 2010). Estas cenizas son un tipo de adsorbente muy versátil debido a sus múltiples propiedades (Swarnalakshmi, 2018), dentro de las cuales se consideran importantes el área superficial y la porosidad. Así mismo, contribuyen a la eliminación de diferen- 
tes tipos de contaminantes en fuentes hídricas, cumpliendo con requisitos como un bajo costo de producción, fácil regeneración y una alta capacidad de adsorción, entre otros (Doria-Herrera, Hormaza-Anaguano, \& Gallego-Suárez, 2013; Martínez-Ángel et al., 2010; Prada \& Cortés, 2010; Sierra-Aguilar, 2009).

Las características fisicoquímicas de las cenizas obtenidas dependen de la técnica que se utilice para extraerlas; es decir, están gobernadas por la temperatura empleada para cada caso. Sin embargo, la combustión siempre se realiza en un rango de $550-800{ }^{\circ} \mathrm{C}$, en donde se ha descubierto que la sílice amorfa tiende a formarse en mayores proporciones cuando la temperatura es más alta, lo cual permite que las cenizas adquieran propiedades diferentes y tengan un uso final distinto a las demás (Menya, Olupot, Storz, Lubwama, \& Kiros, 2018).

La adsorción es un método eficaz para la remoción de metales pesados y otros contaminantes orgánicos de las fuentes hídricas, siendo el carbón activado uno de los materiales adsorbentes más utilizados para tal fin. No obstante, debido al alto costo del carbón activado, en las últimas décadas se ha investigado la posibilidad de utilizar diferentes residuos industriales para la producción de materiales con características adsorbentes. El proceso de adsorción consiste en la fijación de moléculas en la superficie de un sólido por medio de fuerzas de atracción intermoleculares en los centros activos del material estudiado y el sustrato. La adsorción puede ser física o química dependiendo de la unión de las moléculas. En el caso de la adsorción física, la unión se da por medio de fuerzas de van-derWaals, por lo cual la estructura de las moléculas no se ve afectada, mientras que la adsorción química ocurre por atracciones electrostáticas en donde la distribución electrónica en las moléculas es objeto de modificaciones. La adsorción química se caracteriza por ser mucho más lenta que la física debido a que requiere un tiempo determinado para llegar al estado estacionario en función de la presión y la temperatura del proceso, junto con las propiedades químicas del sistema con el cual se está trabajando (Yates, 2011).

Existen dos teorías que describen el proceso de adsorción. La primera, denominada teoría del cubrimiento de superficie, se puede aplicar a nivel general, mientras que la segunda, llamada teoría del llenado de poro, es solamente aplicable para solidos microporosos. Se considera además que el sólido en el cual se adsorben las moléculas se denomina "adsorbente", cuya superficie puede ser de naturaleza homogénea o heterogénea (Klose et al., 2010). En consecuencia, la capacidad de adsorción de un material depende de su superficie específica, de la forma y distribución de la porosidad y de los grupos presentes en la superficie del material a estudiar, principalmente. En el proceso de adsorción se producen variaciones de entalpía del orden de las reacciones de condensación o de cristalización. Por tanto, pequeñas variaciones de temperatura no alteran apreciablemente el fenómeno de adsorción. Por este motivo, los experimentos de adsorción suelen ser realizados a temperatura ambiente, con el consiguiente ahorro energético. Adicionalmente, las propiedades finales del adsorbente están determinadas, en gran medida, por el material precursor que se utilice, debido a que este constituye en forma esencial las características estructurales del material, así como de los procesos fisicoquímicos de activación que se realicen con el objetivo de optimizar su capacidad adsorbente (Shamsollahi \& Partovinia, 2019).

En términos generales, un proceso de activación consiste en abrir poros obstruidos por diferentes tipos de sustancias, manteniendo la estructura original del material de partida. Es así como cada material poroso, dependiendo de la activación que se realice, tendrá una estructura diferente y, por tanto, la ubicación y el tamaño de sus poros será también variable; lo cual guarda relación directa con la capacidad de adsorción del material con el cual se está trabajando (Vargas, Moreno, \& Giraldo, 2008). Básicamente, es un proceso que transcurre generalmente en dos etapas: (i) una que consiste en una impregnación química del material precursor con una disolución concentrada de un agente químico activante y (ii) otra en la que se somete a la mezcla resultante a un proceso pirolítico en el cual se produce simultáneamente la carbonización y la activación del material. El agente químico activante altera el curso de la pirólisis y promueve la deshidratación del material con el aumento de la temperatura, induciendo a la aromatización del material utilizado. El rango de temperaturas empleado es inferior al de los procesos de activación física, el cual varía entre 400 y 900 ㅇ. $\mathrm{C}$. Además, el material resultante es sometido a un proceso de lavado que busca eliminar restos del agente activante, por lo que la porosidad resulta accesible. 
Entre las ventajas que presenta la activación química frente a la activación física se considera: el uso de menores temperaturas de pirólisis, una sola etapa de ejecución, un mayor rendimiento del material obtenido, un mejor desarrollo de la microporosidad y la obtención de productos de superficie específica muy elevada. Entre los agentes químicos utilizados para este tipo de activación, los más utilizados son $\mathrm{ZnCl}_{2}, \mathrm{H}_{3} \mathrm{PO}_{4}$ y $\mathrm{KOH}$. Los dos primeros son agentes deshidratantes que producen un hinchamiento de las partículas durante el proceso de impregnación, ya que la degradación de la celulosa y la lignina es catalizada por ácidos (Martínez-Fierro, 2012).

Aunque los tres agentes químicos producen un gran desarrollo de la micro porosidad, existen diferencias entre ellos: el agente $\mathrm{KOH}$ produce un ensanchamiento del tamaño de los microporos, el $\mathrm{ZnCl}_{2}$ una microporosidad de tamaño uniforme y un menor desarrollo de la mesoporosidad y el $\mathrm{H}_{3} \mathrm{PO}_{4}$ conduce a una distribución de tamaño de poros más heterogénea; este último es comúnmente empleado para activar químicamente residuos orgánicos. Además, durante el proceso de impregnación se produce un aumento de la elasticidad de las partículas debido a que el ácido separa las fibras celulósicas, produciendo una despolimerización parcial de la hemicelulosa y la lignina que conduce a un hinchamiento de la partícula y a una disminución de la resistencia mecánica del precursor (Martínez-Fierro, 2012).

\section{MATERIALES Y MÉTODOS}

\section{Material de Partida}

La CA utilizada para la realización de esta investigación provenía del molino de arroz de la compañía DiCorp (arroz Diana), ubicado en la ciudad de El Espinal, en el departamento del Tolima, Colombia.

\section{Tratamiento previo del material}

Para esta fase del experimento, 50 gramos de CA fueron sometidos a un lavado con $11 \mathrm{ml}$ de ácido clorhídrico diluido en $500 \mathrm{ml}$ de agua para remover impurezas presentes sobre el material. Luego de a alcanzar el punto de ebullición, la solución permaneció 30 minutos más a esta temperatura, tras lo cual se suspendió el calentamiento. Posteriormente, se realizaron va- rios lavados con agua destilada hasta alcanzar un $\mathrm{pH}$ neutro. Finalmente, se realizó una calcinación a 700 ${ }^{\circ} \mathrm{C}$ durante una hora en atmósfera de oxígeno. A este material se le denomina blanco (CB).

\section{Obtención de cenizas activadas con ácido fosfórico}

A 50 gramos de CA, previamente lavada y secada, se adicionaron $500 \mathrm{ml}$ de agua con ácido fosfórico. La relación entre la cantidad de ácido y cascarilla adicionada se realizó en tres cantidades diferentes, según lo descrito por Sarmiento et al., (2004). Las relaciones seleccionadas cascarilla de arroz: ácido a caso fueron 1:1, 1:2 y 1:3, de acuerdo con lo sugerido en trabajos previos (Fierro et al., 2010). Luego de establecer la relación a utilizar, se adicionó la cantidad de ácido correspondiente al agua y junto a la cascarilla se realizó un calentamiento de la mezcla a $90^{\circ} \mathrm{C}$ por 30 minutos. Posteriormente, la muestra se dejó enfriar para luego ser filtrada y calcinada a $700{ }^{\circ} \mathrm{C}$ por una hora. Pasado este tiempo, la muestra fue retirada de la mufla y enfriada, tras lo cual se procedió a molerla y tamizarla para lograr un tamaño de partícula menor de $150 \mu \mathrm{m}$. La tabla 1 presenta la nomenclatura de las muestras utilizadas.

Tabla 1. Nomenclatura de los materiales

\begin{tabular}{cc}
\hline Nombre & Tratamiento \\
\hline CB & Blanco de muestra, cenizas sin activar \\
\hline CA1 & Cenizas Activadas con ácido fosfórico, relación 1:1 \\
\hline CA2 & Cenizas Activadas con ácido fosfórico, relación 1:2 \\
\hline CA3 & Cenizas Activadas con ácido fosfórico, relación 1:3 \\
\hline & Fuente: elaboración propia.
\end{tabular}

\section{Caracterización de los materiales}

\section{Análisis próximo}

El material de partida fue analizado por este método termogravimétrico con el fin de determinar su contenido de humedad, material volátil, contenido de cenizas y carbón fijo (ASTM D3172, 2013).

\section{Número de yodo}

Esta prueba fue realizada con el objetivo de evaluar la capacidad adsorbente que presentan los materiales y 
está relacionada principalmente con la medida de su microporosidad (ASTM D 4607-94, 2006).

\section{Caracterización textural}

Con el fin de caracterizar la superficie de los sólidos, determinar su área superficial y su porosidad, las isotermas de adsorción-desorción de nitrógeno a $77 \mathrm{~K}$ fueron registradas en un equipo TriStar 3000 Micrometrics Surface Area and Porosity Analyser. Previamente a la realización del análisis, las muestras calcinadas fueron desgasificadas durante 4 horas a $200^{\circ} \mathrm{C}$ bajo una atmósfera de nitrógeno. En la determinación del área superficial se empleó la metodología Brunauer-Emmett-Teller (BET). Además, las distribuciones del tamaño de poro fueron elaboradas de acuerdo con el método Barret-Joyner-Halenda [BJH] (Barret \& Joyner, 1951).

\section{Adsorción con azul de metileno (MB, methylene blue)}

La adsorción del ion 3,7 bis (dimetilamino) fenoltiazin 5-ionio, más conocido como azul de metileno (MB por sus siglas en inglés), es un ensayo que se utiliza para evaluar la mesoporosidad del material (Yates, 2011). Se realizaron tres pruebas con cada una de las muestras a una temperatura de $25^{\circ} \mathrm{C}, \mathrm{pH} 3$ y pH 9 y concentraciones de 100,200 y 400 ppm, las cuales se cuantificaron con un espectrofotómetro UV-VIS marca Tuner (Benadjemia, Millière, Reinert, Benderdouche, \& Duclaux, 2011).

La cantidad adsorbida de MB en el equilibrio sobre los materiales $\left(q_{e}\right.$ en $\left.\mathrm{mg} / \mathrm{g}\right)$ fue calculada a partir de la siguiente ecuación:

$$
\mathrm{q}_{\mathrm{e}}=\left[\left(\mathrm{C}_{\mathrm{o}}-\mathrm{C}_{\mathrm{e}}\right) \mathrm{V}\right] / \mathrm{m}
$$

Donde $C_{o}$ es la concentración inicial de $\mathrm{MB}(\mathrm{mg} / \mathrm{L}), C_{e}$ la concentración de $\mathrm{MB}$ en el equilibrio $(\mathrm{mg} / \mathrm{L}), V$ el volumen de la solución $(100 \mathrm{~mL})$ y $m$ la masa del material adsorbente (g).

Adicionalmente, las isotermas de adsorción de $M B$ se ajustaron por el modelo de Langmuir usando la siguiente ecuación:

$$
\mathrm{C}_{\mathrm{e}} / \mathrm{q}_{\mathrm{e}}=1 /\left(\mathrm{k}_{\mathrm{L}} \mathrm{q}_{\mathrm{m}}+\mathrm{C}_{\mathrm{e}} / \mathrm{q}_{\mathrm{m}}\right)
$$

Donde $k_{L}$ es la constante de Langmuir y $q_{m}(\mathrm{mg} / \mathrm{g})$ la cantidad máxima de MB que puede ser adsorbida.
De otro lado, la cantidad máxima de MB adsorbido permite hacer una estimación del área superficial cubierta por la molécula $A M\left(S_{M B}\right)$ a partir de la siguiente ecuación:

$$
S_{M B}=q_{m} \times A_{m} \times 6.02 * 10^{23} / M_{M B} \quad \text { (Ec. 3) }
$$

Lo anterior da como resultado una superficie molecular para el $\mathrm{MB}\left(\mathrm{A}_{\mathrm{m}}\right)$ de $1,30 \mathrm{~nm}^{2}$ y un peso molecular ( $\mathrm{M}_{\mathrm{MB}}$ ) de $284 \mathrm{~g} / \mathrm{mol}$ (Benadjemia et al., 2011).

\section{Microscopía electrónica de barrido}

El experimento incorporó la realización de microscopía electrónica de barrido con el fin de observar la morfología de las cenizas de manera directa. A través de esta técnica, un haz de electrones en el vacío provoca la excitación de electrones secundarios en la muestra, los cuales envían señales que son captadas como una imagen (Souza-Macedo et al., 2006).

\section{Espectroscopía de infrarrojo}

Se utilizó un espectrofotómetro infrarrojo con transformada de Fourier, Argileent CARY 630, en el que se analizaron las muestras sólidas secas realizando un barrido entre los 4000 y $200 \mathrm{~cm}^{-1}$. Esta técnica se basa en la capacidad de absorción de la radiación infrarroja de cualquier sustancia para determinar los grupos funcionales presentes en el material evaluado. Por tanto, esta se realizó para conocer no solo los grupos funcionales de las cenizas, sino también para evaluar los cambios a partir del proceso de activación.

\section{RESULTADOS Y DISCUSIÓN}

\section{Caracterización por análisis próximo}

Al realizar el análisis próximo según la norma ASTM D3172, se concluye que el material obtenido a partir de la calcinación de CA está conformado por los siguientes componentes: humedad $(16,7 \%)$, materia volátil $(19,9 \%)$, cenizas $(35,9 \%)$ y carbono fijo $(27,5$ $\%)$. Por lo anterior, se puede afirmar que el sólido con el cual se está trabajando es una mezcla de diversos componentes en cuya superficie se encuentra un considerable porcentaje de carbono fijo, lo cual le brinda al material excelentes propiedades como adsorbente. Así mismo, en mayor porcentaje se encuentra la ceniza, razón por la que el material no se considera un 
carbón sino una mezcla de cenizas que luego del proceso de activación serán denominadas cenizas activadas.

\section{Número de yodo}

El objetivo principal de esta prueba es identificar la cantidad de yodo adsorbido en la superficie, el cual es una aproximación del área superficial del material carbonáceo. Los resultados muestran la capacidad para adsorber moléculas de diámetro pequeño con características no polares en todos los materiales. Los números de yodo se presentan en la tabla 2 a continuación.

Tabla 2. Adsorción de yodo

\begin{tabular}{cc}
\hline Muestras & Número de yodo $(\mathbf{m g} / \mathbf{g})$ \\
\hline CB & 826,0 \\
\hline CA1 & 260,5 \\
\hline CA2 & 416,9 \\
\hline CA3 & 420,0 \\
\hline
\end{tabular}

Fuente: elaboración propia.

Al comparar los resultados se puede observar que los números de yodo obtenidos para las cenizas activadas con ácido fosfórico son menores que para $\mathrm{CB}$, hecho que puede ser atribuido a una distribución de tamaño de poro heterogénea luego de la activación, en la cual la cantidad de microporos disminuyó drásticamente después de la calcinación, ocasionando dificultades en la inclusión de las moléculas de yodo sobre la superficie de las cenizas. Además, es posible que los microporos tiendan a colapsar cuando la temperatura de calcinación se enfría, haciendo inaccesible la entrada a estos a temperatura ambiente. Sin embargo, respecto a las cenizas activadas, el número de yodo aumenta proporcionalmente con la cantidad de ácido utilizada en la activación, manifestando un efecto benéfico de esta activación en el área superficial.

\section{Caracterización textural}

La figura 1 presenta las isotermas de adsorción-desorción de nitrógeno de los materiales. En general, se observa una mayor adsorción a presiones relativas altas, la cual es característica de los sólidos macroporosos. No obstante, se puede apreciar que la parte siguiente de la isoterma no es lineal, lo que indica la continuación del proceso de adsorción a presiones relativas mayores como consecuencia de la presencia de una mesoporosidad desarrollada.

Figura 1. Isotermas de adsorción de nitrógeno en los materiales adsorbentes

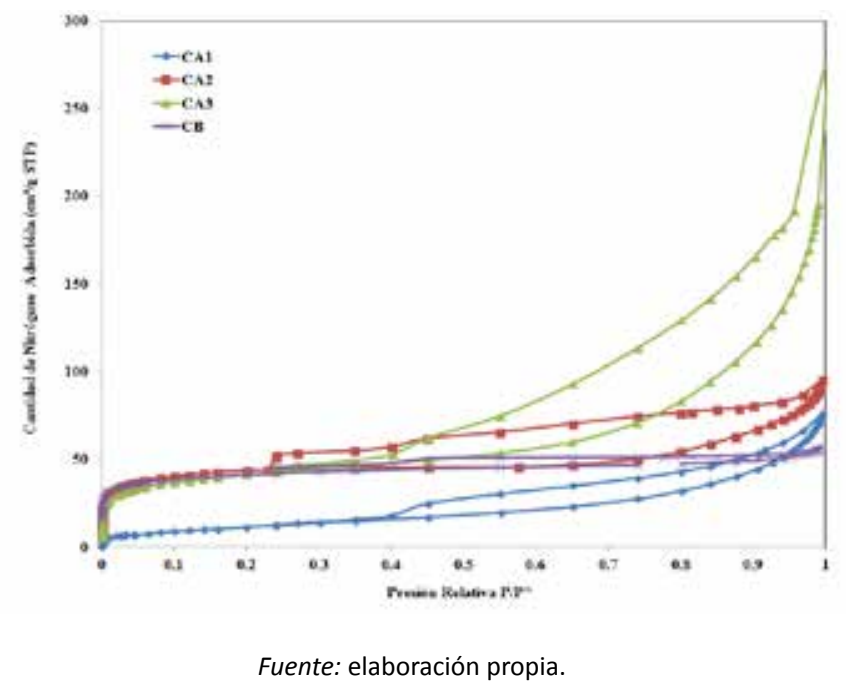

Las isotermas obtenidas en los sólidos corresponden a isotermas tipo IV, las cuales tienen como rasgo distintivo su lazo de histéresis según la calificación BDDT (Brunauer, Deming, Deming, \& Teller, 1940) (Yates, 2011). En la mayoría de los casos, las isotermas presentan histéresis tipo H3, de acuerdo con la clasificación de la Unión Internacional de Química Pura y Aplicada (IUPAC, en inglés), y corresponden al tipo de histéresis más común exhibido por la mayoría de óxidos inorgánicos. Este tipo de histéresis es usual en sólidos que consisten de agregados o aglomerados de partículas que forman poros en forma de ranuras. Ejemplos típicos de este tipo de isotermas son las zeolitas y los carbones activados.

De otro lado, esta clase de isoterma es característica de adsorbentes meso y macroporosos, lo que concuerda con los resultados obtenidos con el número de yodo en los que la cantidad de microporos es baja. Respecto al área superficial estimada por el método $B E T$, se puede observar el efecto positivo en la activación con ácido dado en los sólidos CA1, CA2 y CA3, en los que se evidencia un aumento en el área superficial respecto al material CB. En términos generales, las diferentes muestras obtenidas durante el proceso presentan una distribución de poros heterogénea. Sin embargo, la tabla 3 presenta una medida promedio de los tamaños de poro que varía dependiendo de la ejecución de una activación previa al material, lo cual 
beneficia los procesos que requieren adsorción de moléculas con diferentes tipos de tamaños.

Tabla 3. Características texturales de los materiales

\begin{tabular}{ccc}
\hline Material & Área superficial BET $\left(\mathbf{m}^{2} / \mathbf{g}\right)$ & Tamaño de poro $(\mathbf{n m})$ \\
\hline $\mathrm{CB}$ & 141 & 4,0 \\
\hline $\mathrm{CA} 1$ & 160 & 9,0 \\
\hline $\mathrm{CA} 2$ & 225 & 14,0 \\
\hline $\mathrm{CA} 3$ & 312 & 12,0 \\
\hline
\end{tabular}

Fuente: elaboración propia.

\section{Adsorción con azul de metileno (MB)}

El MB es una molécula orgánica bastante grande, heterocíclica y soluble en agua que es apropiada para ensayos de adsorbentes en materiales cuya mesoporosidad sugiere su aplicación para la adsorción de contaminantes en fase líquida. Esta molécula tiene la siguiente fórmula molecular: $\left(\mathrm{C}_{16} \mathrm{H}_{18} \mathrm{~N}_{3} \mathrm{~S}\right)^{+} \mathrm{Cl}$. Cuando se disuelve en agua su tamaño molecular es de 0,7 $\mathrm{nm} \times 1,6 \mathrm{~nm}$ basado en estimaciones Van der Waals. Se espera que la forma hidratada sea aún más grande, lo que facilitaría la afinidad por los mesoporos de carbono que poseen centros activos aromáticos (Fierro et al., 2010).

Los parámetros del modelo de Langmuir aplicado a la adsorción de $\mathrm{MB}$ en los materiales adsorbentes a $\mathrm{pH}$ 3 y 9 fueron calculados de acuerdo con las ecuaciones (1), (2) y (3), cuyos resultados se presentan a continuación en las tablas 4 y 5 , respectivamente.

Tabla 4. Parámetros del modelo de Langmuir aplicado a la adsorción de azul de metileno en los materiales adsorbentes a pH = 3 y su relación con el área externa calculada por el método BET (SBET)

\begin{tabular}{ccccc}
\hline \multirow{2}{*}{ Muestra } & \multicolumn{2}{c}{ Parámetros del modelo de Langmuir } & Área cubierta por MB & Relación SMB/sBET \\
\cline { 2 - 5 } & $\mathrm{qm}(\mathrm{mg} / \mathrm{g})$ & $\mathrm{kL}(\mathrm{L} / \mathrm{g})$ & $\mathrm{SMB}(\mathrm{m} 2 / \mathrm{g})$ & $(\%)$ \\
\hline CA1 & 64,5 & 0,19 & 178 & $>100$ \\
$\mathrm{CA} 2$ & 75,8 & 0,04 & 209 & 93 \\
$\mathrm{CA} 3$ & 153,8 & 0,07 & 424 & $>100$ \\
\hline
\end{tabular}

Fuente: elaboración propia.

Tabla 5. Parámetros del modelo de Langmuir aplicado a la adsorción de azul de metileno en los materiales adsorbentes a pH = 9 y su relación con el área externa calculada por el método BET $\left(\mathrm{S}_{\mathrm{BET}}\right)$

\begin{tabular}{ccccc}
\hline \multirow{2}{*}{ Muestra } & \multicolumn{2}{c}{ Parámetros del modelo de Langmuir } & Área cubierta por MB & Relación $\mathrm{S}_{\mathrm{MB}} / \mathrm{SBET}$ \\
\cline { 2 - 5 } & $\mathrm{q}_{\mathrm{m}}(\mathrm{mg} / \mathrm{g})$ & $\mathrm{k}_{\mathrm{L}}(\mathrm{L} / \mathrm{g})$ & $\mathrm{S}_{\mathrm{MB}}\left(\mathrm{m}^{2} / \mathrm{g}\right)$ & $(\%)$ \\
\hline CA1 & 38,2 & 0,01 & 105 & 65 \\
\hline $\mathrm{CA} 2$ & 39,2 & 0,01 & 109 & 48 \\
\hline CA3 & 87,7 & 0,03 & 242 & 78 \\
\hline
\end{tabular}

Fuente: elaboración propia.

De manera general, se puede apreciar que la capacidad de adsorción de MB $\left(q_{m}\right)$ en los materiales obtenidos a partir de CA aumenta con el área superficial $\mathrm{S}_{\mathrm{BET}}$ calculada mediante las isotermas de adsorción de nitrógeno. La mayor $\mathrm{q}_{\mathrm{m}}$ fue estimada para el material CA3 a un pH de 3,154 mg/g. La relación entre el área superficial cubierta por el $\mathrm{MB}\left(\mathrm{S}_{\mathrm{MB}}\right)$ y el área superficial BET ( $\mathrm{S}_{\mathrm{BET}}$ ) es un indicativo de la capacidad del MB para alcanzar los micro y los mesoporos del material. En este sentido, los resultados indican que esta capacidad de cubrimiento es más elevada a un $\mathrm{pH}$ de 3. Así mismo, señalan que los poros del material tienen un tamaño mayor a $1,5 \mathrm{~nm}$, lo cual concuerda no solamente con los resultados obtenidos a partir del análisis textural (tabla 3) sino con trabajos como ele de Graham (1955), quien sugiere que 1,33 $\mathrm{nm}$ es el tamaño de poro requerido para que la adsorción pueda ocurrir. 


\section{Microscopía electrónica de barrido}

Las imágenes SEM de uno de los materiales exhiben una superficie heterogénea porosa y con crecimiento laminar, como se muestra en la figura 2. Así mismo, de estas imágenes puede estimarse tamaños de partícula entre 300 y $500 \mu \mathrm{m}$.

Figura 2. Imágenes SEM de un material adsorbente obtenido a partir de la cascarilla de arroz
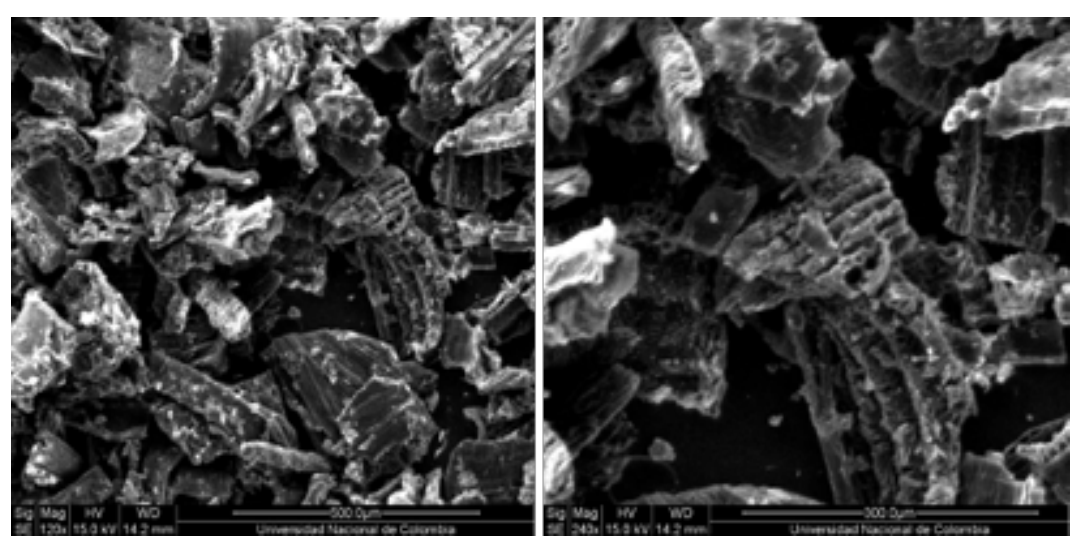

Fuente: elaboración propia.

\section{Espectroscopía de infrarrojo}

La figura 3 muestra el cambio en las bandas de absorción en el espectro IR de los materiales activados respecto al material CB.

Figura 3. Espectros infrarrojos de las muestras CB y de una muestra activada

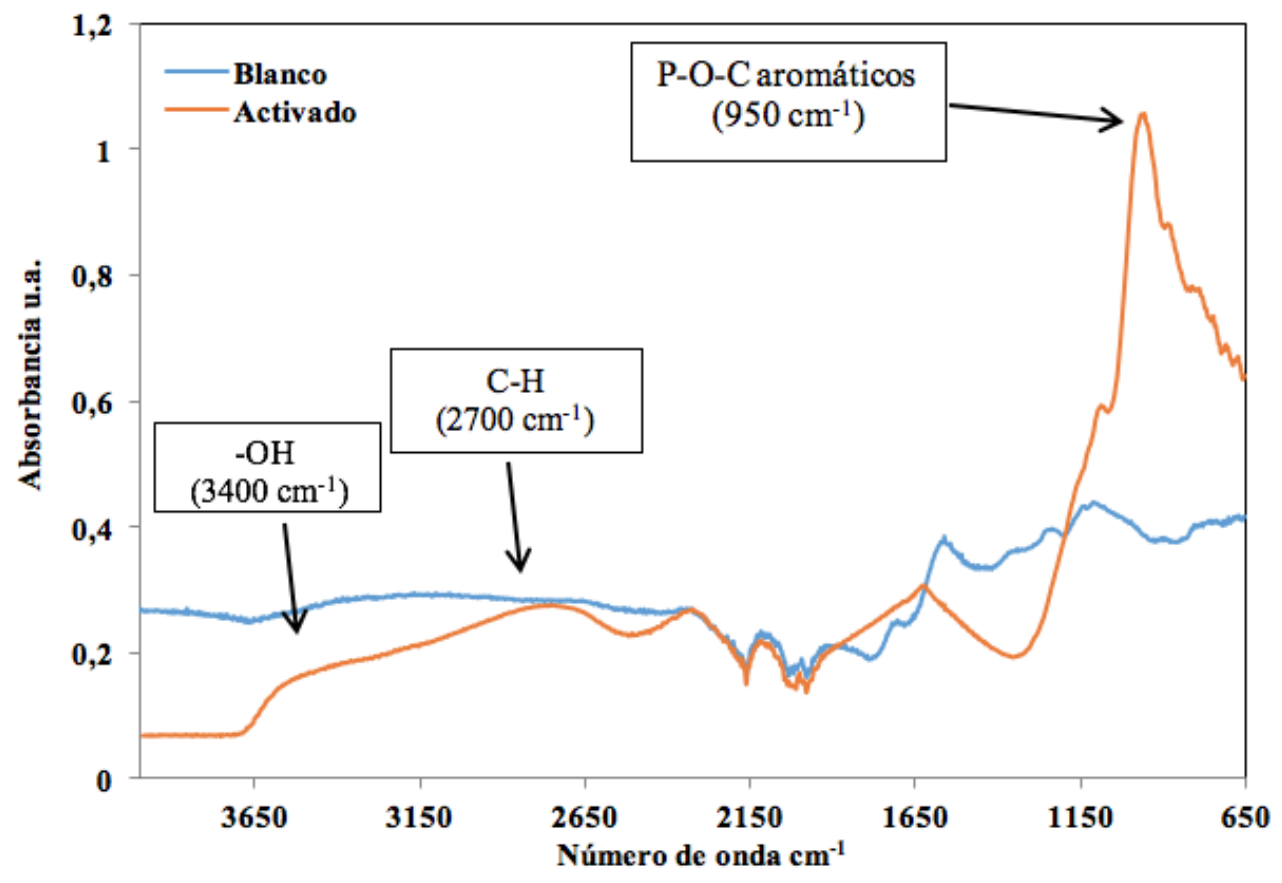

Fuente: elaboración propia. 
Una amplia banda de absorción entre 3200 y 3600 $\mathrm{cm}^{-1}$ con un máximo alrededor de $3400 \mathrm{~cm}^{-1}$ es característica de las vibraciones de estiramiento de los grupos hidroxilo (grupos carboxilo, fenoles y alcoholes), además de agua adsorbida en la superficie. Esta banda es mayor y mejor definida en el sólido después de la activación con ácido. Así mismo, en el sólido activado se define una banda en $2700 \mathrm{~cm}^{-1}$ relacionada con la vibración de estiramiento del enlace $\mathrm{C}-\mathrm{H}$ (en grupos aromáticos, grupos metilo y metileno de cadenas laterales).
Alrededor de $1100 \mathrm{~cm}^{-1}$ se observa la aparición de una banda en la muestra activada con ácido. Esta banda usualmente está relacionada con carbonos oxidados y ha sido asignada al estiramiento $\mathrm{C}-\mathrm{O}$ en ácidos, alcoholes, fenoles, éteres o esteres. Sin embargo, una asignación precisa no es posible debido a que en esta misma región se encuentran asignadas las vibraciones de estiramiento de los enlaces P-O-C. Además, el pequeño hombro en $1100 \mathrm{~cm}^{-1}$ ha sido relacionado con el enlace $\mathrm{P}-\mathrm{O}$ en esteres ácidos fosfato y para la vibración simétrica en la cadena P-O-P (Benadjemia et al., 2011).

Figura 4. Espectros infrarrojos de las muestras activadas.

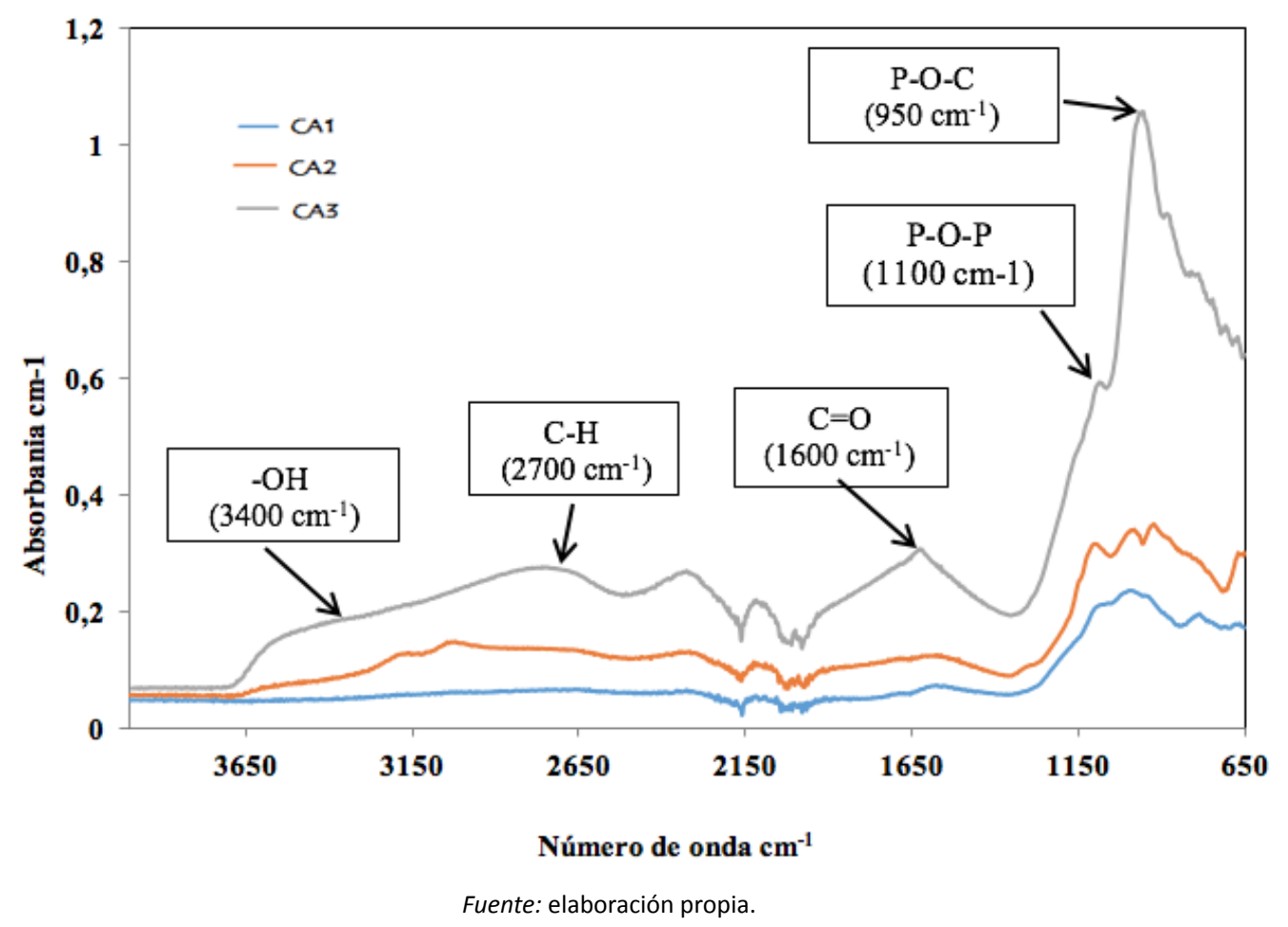

La figura 4 permite observar los espectros IR de las muestras activadas en relación a la proporción de ácido utilizada durante la activación 4 . El aumento en la absorción en la región entre 3200 y $3600 \mathrm{~cm}^{-1}$ (relacionada con la vibración de los grupos $\mathrm{OH}$ ) y de la banda en $1600 \mathrm{~cm}^{-1}$ (relacionada con la vibración del enlace $\mathrm{C}=\mathrm{O}$ ) son cambios evidentes ocasionados por el aumento en la concentración del ácido utilizada durante la activación que generan un aumento en la adsorción de moléculas de agua en la superficie y del grupo de carbonos oxidados, respectivamente.
En relación con la proporción de ácido, el aumento proporcional de la banda de adsorción en $950 \mathrm{~cm}^{-1}$ en relación con la concentración del ácido, permite hacer de una forma más precisa una asignación de dicha banda a la vibración del grupo P-O-C y del hombro en $1100 \mathrm{~cm}^{-1}$ con el grupo P-O-P. Para concluir con la caracterización IR, el otro cambio significativo a nivel estructural con el aumento en la proporción de ácido usada durante la activación, es el desarrollo de la vibración $\mathrm{C}-\mathrm{H}\left(2700 \mathrm{~cm}^{-1}\right)$, el cual se debe, posiblemente, a la pérdida de oxígeno en la superficie del material carbonáceo. 


\section{CONCLUSIONES}

Los materiales absorbentes obtenidos a partir del tratamiento químico de las cenizas de cascarilla de arroz evidenciaron un aumento en el valor del área superficial al compararlos con las cenizas obtenidas bajo las mismas condiciones pero sin ningún tratamiento químico. Dado que el área superficial está directamente relacionada con la porosidad total de la muestra, este aumento se debe a la contribución de poros que se han formado como resultado de la activación química, lo que confirma que el tratamiento aumenta el área superficial y el diámetro medio de poro.

Los materiales adsorbentes mostraron un área superficial cercana a $320 \mathrm{~m}^{2} / \mathrm{g}$, la cual disminuye directamente dependiendo de la cantidad de ácido usado en la activación. Los tamaños de poro de las muestras trabajadas oscilan entre 4 y $12 \mathrm{~nm}$, indicando que se obtuvieron materiales mesoporosos.

De manera global, se puede concluir que la capacidad de adsorción de $M B\left(q_{m}\right)$ en los materiales obtenidos a partir de la cascarilla de arroz aumenta con el área superficial $S_{B E T}$ calculada mediante las isotermas de adsorción de nitrógeno. La mayor $\mathrm{q}_{\mathrm{m}}$ fue estimada para el material CA3 a un $\mathrm{pH}$ de 3.

Los resultados de este trabajo evidencian que el proceso de producción de las cenizas activadas a partir de la cascarilla de arroz y su uso como materiales adsorbentes es viable, señalando así una nueva oportunidad de aprovechamiento para materiales considerados como desechos agrícolas.

\section{REFERENCIAS}

Areiza-Segura, A. M. (2012). Diagnóstico del mercado del arroz en Colombia (2000-2012). Bogotá. Superintendencia de Industria y Comercio. Colombia

ASTM D4607-94. (2006). Standard Test Method for Determination of lodine Number of Activated Carbon. ASTM International.

ASTM D3172. (2013). Standard Practice for Proximate Analysis of Coal and Coke. ASTM International.

Barret, E. P., \& Joyner, L. G. (1951). The Determination of Pore Volume and Area Distributions. J. Am.
Chem. Soc. 73(1), 373-380, doi: https://doi. org/10.1021/ja01145a126

Benadjemia, M., Millière, L., Reinert, L., Benderdouche, N., \& Duclaux, L. (2011). Preparation, characterization and Methylene Blue adsorption of phosphoric acid activated carbons from globe artichoke leaves. Fuel Processing Technology, 92, 1203-1212, doi: https://doi.org/10.1016/j. fuproc.2011.01.014

Carrillo-Quijano, C. C., Albarracín-Caballero, J., \& Pereira-Hernández, X. (2013). Producción de carbón activado y sílice a partir de cascarilla de arrozna revisión. Scientia Et Technica, 18(2), 422429.

Doria-Herrera, G. M., Hormaza-Anaguano, A., \& Gallego-Suárez, D. (2013).Caracterización estructural de la cascarilla de arroz modificada como adsorbente alternativo y eficiente para la remoción de $\mathrm{Cr}(\mathrm{VI})$ en solución. Revista de Investigación Agraria y Ambiental, 4(1), 22-29, doi: https://doi.org/10.22490/21456453.976

Federación Nacional de Industriales del Arroz (2013). Política comercial para el arroz. Documento de la ANDI, Cámara Induarroz, Colombia.

Fierro, V., Muñiz, G., Basta, A. H., El-Saied, H., \& Celzard, A. (2010). Rice straw as precursor of activated carbons: Activation with ortho-phosphoric acid. Journal of Hazardous Materials, 181, 27-34, doi: https://doi.org/10.1016/j.jhazmat.2010.04.062

Leofanti, M. (1998). Surface area and pore texture of catalysts. Catalysis Today, 207-219.Graham, D. (1955). Characterization oh physical adsorption systems III. The separate effects of pore size and surface acidity upon the adsorbent capacities of activated carbons. The Journal of Physical Chemistry, 59(8), 896-900, doi: https://doi. org/10.1016/S0920-5861(98)00050-9

Klose, W., Rincón, S., \& Gómez, A. (2010). Carbón activado de cuesco de palma. Estudio de termogavimetría y estructura. Alemania: Kassel university.

Martínez-Ángel, J. D., Pineda-Vásquez, T. G., LópezZapata, J. P., \& Betancur-Vélez, M. (2010). Fluidized bed rice husk combustion experiments for the 
production of silica-rich ash. Revista Facultad de Ingeniería Universidad de Antioquia, 51, 97-104.

Martínez-Fierro, M. C. (2012). Preparación y caracterización de carbón activo a partir de lignina para su aplicación en procesos de descontaminación de aguas. Facultad de Ciencias, Universidad Autónoma de Madrid, España.

Menya E., Olupot P., Storz H., Lubwama M., Kiros Y. (2018) Production and performance of activated carbon from rice husk for removal of natural organic matter from water: A review. Chemical engineering Research and Design, 129, 271-296, doi: https://doi.org/10.1016/j.cherd.2017.11.008

Prada, A., \& Cortés, C. E. (2010). La descomposición térmica de la cascarilla de arroz: una alternativa de aprovechamiento integral. Revista Orinoquia, 14(Supl. 1), 155-170.

Quinceno-Villada, D., \& Mosquera-Gutiérrez, M. Y. (23 de Abril de 2010). Alternativas tecnológicas para el uso de la cascarilla de arroz como combustible. Universidad Autónoma de Occidente, Santiago de Cali, Colombia.

Sarmiento, C., Sánchez, J., García, C., Rincón, Y., Benítez, A., \& Ramírez, J. (2004). Preparación de carbón activado mediante la activación química de carbón mineral. Ciencia 12(1), 52-63.

Shamsollahi Z., \& Partovinia A. (2019) Recent advances on pollutants removal by rice husk as a bio-based adsorbent: A critical review. Journal of enviromental management, 246, 314-323.

Sierraguila, J. (2009). Alternativas de aprovechamiento de la cascarilla de arroz en Colombia. Universidad de Sucre, Facultad de Ingenería, Sincelejo, Colombia.

Souza-Macedo, J., Bezerra-Costa, N., Almeida, L., Cestari, A., Silva Vieira, E., Gimenez, I., . . . SilvaBarreto, L. (2006). Kinetic and calorimetric study of the adsorption of dyes on mesoporousactivated carbon prepared from coconut coir dust. Journal of Colloid and Interface. Science, 298, 515-522, doi: https://doi.org/10.1016/j.jcis.2006.01.021

Swamalakshmi, K., Prakash C., Nivetha S., \& Athira N. (2018). Use of rice husk ash as an adsorbent to remove contaminants in water and comparison with advanced oxidation process-a study. Materials Today, 5, 24248-24257, doi: https://doi. org/10.1016/j.matpr.2018.10.220

Valverde, A., Sarria L, B., \& Monteaguido, J. P. (2007). Análisis comparativo de las características fisicoquímicas de la cascarilla de arroz. Scientia Et Technica, 1(37), 255-260.

Vargas, J., Moreno, J., \& Giraldo, L. (2008). Obtención y caracterización de carbones activados a partir de semillas de mucuna sp. Revista Colombiana de Química, 37(1), 67-77.

Yates, M. (2011). Área supe ficial, textura y distribución porosa. En Técnicas de Análisis y Caracterización de Materiales. Biblioteca de Ciencias-CSIC. 